\title{
Low-Intensity Pulsed Ultrasound and Halo Immobilization Is an Effective Treatment for Nonunion Following Traumatic Spondylolisthesis of the Axis
}

\author{
Kohtaroh Oda ${ }^{1}$ Tetsuro Ohba ${ }^{1}$ Shigeto Ebata ${ }^{1}$ Hirotaka Haro ${ }^{1}$ \\ ${ }^{1}$ Department of Orthopaedic Surgery, University of Yamanashi, \\ Yamanashi, Japan

\begin{abstract}
Address for correspondence Tetsuro Ohba, MD, PhD, Department of Orthopaedics, University of Yamanashi, 1110, Shimokato, Chuo,
\end{abstract} \\ Yamanashi 409-3898, Japan (e-mail: tooba@yamanashi.ac.jp).
}

J Neurol Surg Rep 2017;78:e119-e122.

\begin{abstract}
This case report describes a unique case involving traumatic spondylolisthesis of the axis that resulted in nonunion, angulation, and displacement after conservative treatment with a cervical collar, but which was successfully achieved union with halo immobilization and low-intensity pulsed ultrasound (LIPUS). Halo immobilization of a traumatic spondylolisthesis in a 20-year-old patient, that previously failed to

Keywords

- nonunion

- traumatic spondylolisthesis of the axis

- low-intensity pulsed ultrasound

- bone union improve after wearing a cervical collar for 3 months, was immediately followed by treatment with a LIPUS device (SAFHS 4000J; Teijin Pharma, Tokyo, Japan) 20 minutes once daily to the right and left fracture sites which were located using fluoroscopic guidance. Radiographs and computed tomography showed conclusive evidence of bone union after 10 weeks of treatment with halo immobilization. No adverse events were observed. To the best of our knowledge, this is the first report describing that the combination of halo immobilization and LIPUS therapy might be a safe, effective, and feasible method by which to treat cervical spine fractures.
\end{abstract}

\section{Introduction}

Traumatic spondylolisthesis of the axis is considered one of the most frequent forms of upper cervical spine injury. The injury comprises 4 to $7 \%$ of all traumatic cervical spine fractures and is classified based on the system described by Effendi et al with modifications proposed by Levine and Edwards (L-E). ${ }^{1}$ Recent studies suggest that based on the L-E system, type I and type II traumatic spondylolisthesis of the axis have been satisfactorily treated, mostly using a conservative approach, which can result in a progressively improved function, a low incidence of neurological deficits, and complications. ${ }^{2,3}$ The majority of cases of traumatic spondylolisthesis of the axis can be treated nonoperatively with reduction and subsequent immobilization in a rigid cervical collar or halo device. For unstable fractures, including some L-E type II and most type IIa/III fractures, or when external brace immobilization is ineffective (for example, in cases of delayed unions or nonunions), surgical management is indicated and can be accomplished by using either anterior or posterior fusion techniques. ${ }^{4}$ Although surgical techniques for traumatic spondylolisthesis of the axis have advanced, problems, such as the risk of neurovascular injury and postoperative complications still remain. ${ }^{5,6}$

A low-intensity pulsed ultrasound (LIPUS) device has been developed for the acceleration of fracture healing. Recent studies have reported the benefits of LIPUS for fresh fractures as well as delayed unions and nonunions. ${ }^{7,8}$ These results suggest that LIPUS could be used as an alternative treatment to surgery.

Herein, we report a unique case of delayed union of a traumatic spondylolisthesis of the axis that was successfully treated with halo immobilization and LIPUS. To the best of our knowledge, this is the first report showing that LIPUS might be a feasible treatment for cervical spine fractures. received

April 20, 2017

accepted

September 20, 2017
DOI https://doi.org/

10.1055/s-0037-1607425. ISSN 2193-6358.
๑) 2017 Georg Thieme Verlag KG
Stuttgart · New York

License terms

(ब(1) $\Theta \circledast$ 


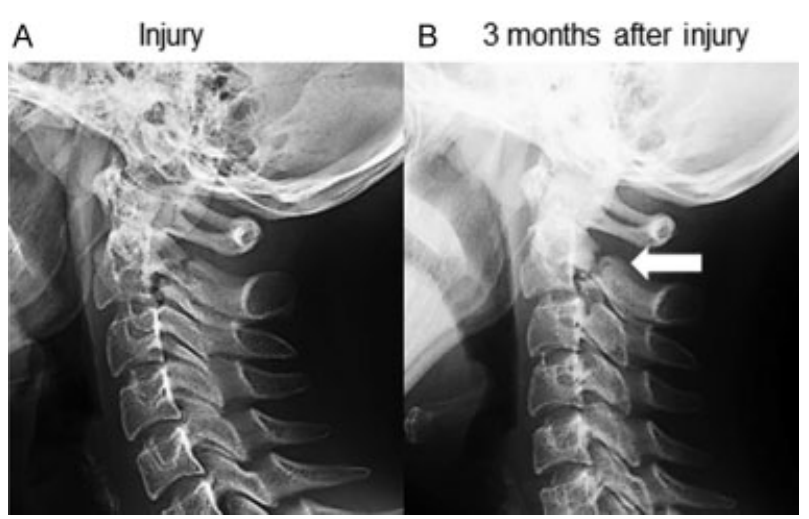

Fig. 1 (A) Cervical plain radiograph at the time of injury and (B) 3 months later. The white arrow denotes delayed union and the development of angulation and displacement.

\section{Case Report}

A 20-year-old woman presented with neck pain after the cervical trauma that occurred during a motor vehicle accident. Plain radiographs and computed tomography (CT) scans of the cervical vertebrae showed type I traumatic spondylolisthesis of the axis using the modified L-E classification. Conservative therapy with a rigid cervical collar was the immediate postinjury treatment used. After 12 weeks of the injury, the patient was referred to our orthopedic department because of nonunion and development of angulation and displacement (-Fig. 1). An initial examination in our department did not reveal the presence of any neurologic deficit. The patient was placed in a neutral position with her head and neck in a halo vest. Immediately after halo immobilization, treatment with a LIPUS device (SAFHS 4000J; Teijin Pharma, Tokyo, Japan) was applied for 20 minutes once daily to the right and left fracture sites after marking the fracture position under fluoroscopic guidance (-Fig. 2). The LIPUS device had a frequency of $1.5 \mathrm{MHz}$, a signal burst width of $200 \mu \mathrm{s}$, a signal repetition

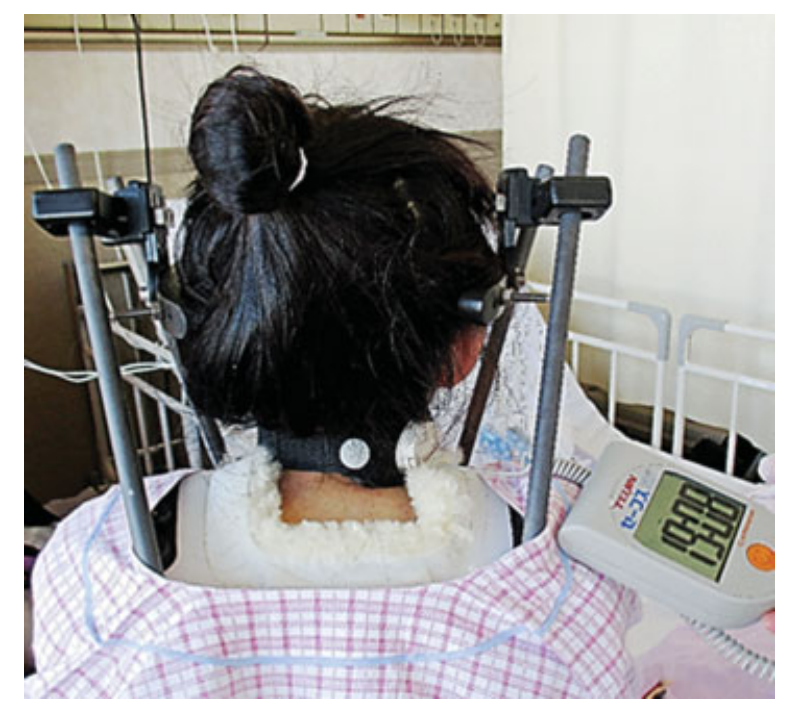

Fig. 2 Macroscopic picture showing low-intensity pulsed ultrasound being used as an adjuvant therapy after halo immobilization. frequency of $1 \mathrm{kHz}$, and an intensity of $30 \mathrm{~mW} / \mathrm{cm}^{2}$. Radiographs and a CT scan showed improved healing of the fracture 3 and 10 weeks after the initiation of LIPUS (-Fig. $\mathbf{3}$ ). After 11 weeks of starting LIPUS was started, the halo vest was removed, and the patient had structurally and functionally recovered. The clinical follow-up at 12 months revealed no symptoms, such as neck pain and discomfort, which suggest pseudarthrosis. Because the application of LIPUS for spinal fractures is considered to be an off-label use of this device, it was approved by the ethical committee of Yamanashi University before LIPUS treatment was started (approval number: 152).

\section{Discussion}

Both delayed and nonunions can lead to additional suffering, and prolonged functional impairment for patients, as well as increased health care system costs. ${ }^{9}$ Delayed unions, and nonunions often require additional complex surgical procedures to heal. ${ }^{10}$ In the current case, 3 months of conservative therapy using a cervical collar failed to prevent increasingly severe angulation and displacement indicative of instability at the fracture site. For unstable fractures that are the result of traumatic spondylolisthesis of the axis (including some L-E type II and most type IIa/III fractures, or when external brace immobilization is ineffective), surgical management is indicated. ${ }^{1}$

When the decision to proceed with surgical fixation has been made, the various surgical techniques suggested include a posterior approach, an anterior approach or a combined anterior and posterior approach. ${ }^{11}$ The anatomy of the upper cervical spine has large individual variations, and the presence of surrounding neurovascular structures makes pedicle screw fixations even more technically challenging. Misplacement and complications of pedicle screws placed using fluoroscopic techniques have been reported in up to $21.6 \%$ of cervical trauma patients. ${ }^{12}$ In contrast, the disadvantage of conservative treatment with the halo device is prolonged immobilization for an additional 3 to 6 months with an uncertain outcome. Therefore, after halo immobilization, and before any decision being made to proceed with surgical fixation, we proposed the use of LIPUS as an adjuvant therapy and obtained an excellent outcome. We could see evidence of bone union only 10 weeks after the initiation of conservative treatment using halo immobilization. Importantly, we did not observe any adverse events.

It has been reported that clinical success rates with LIPUS for delayed unions and nonunions in long bones can range from 67 to $90 \% .^{13}$ Interestingly, a positive effect of LIPUS on spinal fusion has been demonstrated in several animal experiments. ${ }^{8,14,15}$ However, clinical data are completely lacking with respect to the efficacy and safety of LIPUS for spinal fractures in humans. Despite this, based on the current case we cannot conclude LIPUS alone can achieve a successful union in the case of previously delayed unions and nonunions of traumatic spondylolisthesis of the axis, but these results might indicate the combination of halo immobilization and LIPUS can synergistically induce bone union. 
A
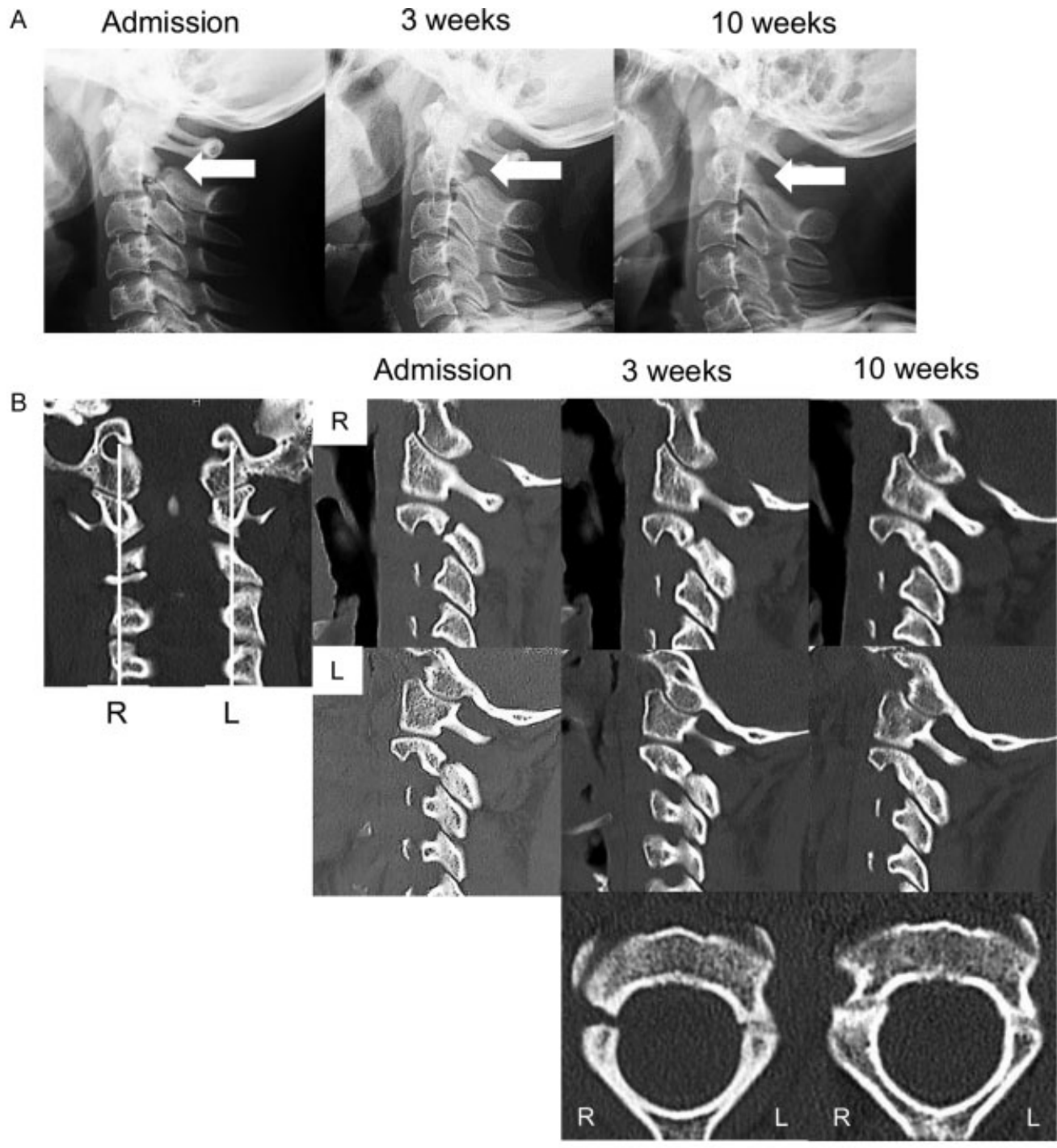

C

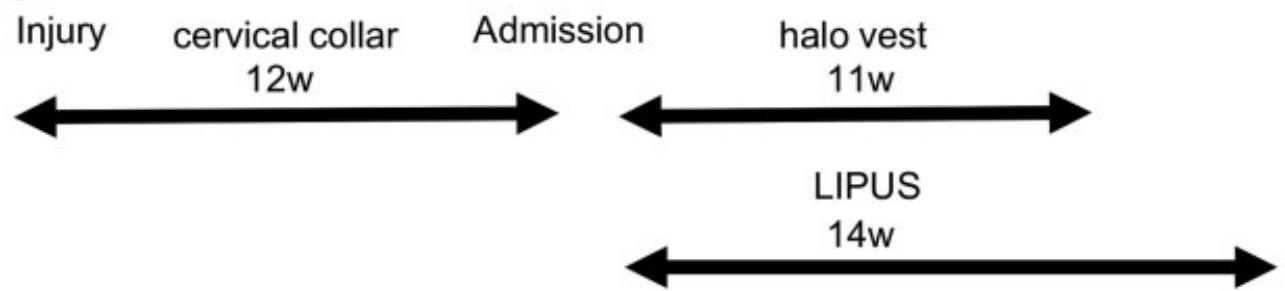

Fig. 3 (A) Radiographs with an arrow showing the C2 vertebra at admission, 3 weeks after initiation of low-intensity pulsed ultrasound (LIPUS), and 10 weeks after initiation of LIPUS. (B) Computed tomography (CT) scans showing, lateral (right and left side) views at admission, 3 weeks after initiation of LIPUS, and 10 weeks after initiation of LIPUS, and axial views (bottom two panels) of C2 vertebra at 3 and 10 weeks after the initiation of LIPUS. White lines indicate the locations of the sagittal sections. Favorable healing of the fracture can be seen on the radiographs, and CT scans taken after initiation of LIPUS. (C) Time series of treatments was shown.

To the best of our knowledge, this is the first report describing that the combination of halo immobilization and LIPUS therapy might be a safe, effective, and feasible method by which to treat cervical spine fractures.

Conflict of Interest

All authors confirm that there are no conflicts of interest.

\section{References}

1 Levine AM, Edwards CC. The management of traumatic spondylolisthesis of the axis. J Bone Joint Surg Am 1985;67(02):217-226

2 Ferro FP, Borgo GD, Letaif OB, Cristante AF, Marcon RM, Lutaka AS. Traumatic spondylolisthesis of the axis: epidemiology, management and outcome. Acta Ortop Bras 2012;20(02):84-87

3 Müller EJ, Wick M, Muhr G. Traumatic spondylolisthesis of the axis: treatment rationale based on the stability of the different fracture types. Eur Spine J 2000;9(02):123-128 
4 Li XF, Dai LY, Lu H, Chen XD. A systematic review of the management of hangman's fractures. Eur Spine J 2006;15(03):257-269

5 Buchholz AL, Morgan SL, Robinson LC, Frankel BM. Minimally invasive percutaneous screw fixation of traumatic spondylolisthesis of the axis. J Neurosurg Spine 2015;22(05):459-465

6 Singh PK, Garg K, Sawarkar D, et al. Computed tomographyguided C2 pedicle screw placement for treatment of unstable hangman fractures. Spine 2014;39(18):E1058-E1065

7 Gebauer D, Mayr E, Orthner E, Ryaby JP. Low-intensity pulsed ultrasound: effects on nonunions. Ultrasound Med Biol 2005; 31(10):1391-1402

8 Romano CL, Romano D, Logoluso N. Low-intensity pulsed ultrasound for the treatment of bone delayed union or nonunion: a review. Ultrasound Med Biol 2009;35(04):529-536

9 Heckman JD, Sarasohn-Kahn J. The economics of treating tibia fractures. The cost of delayed unions. Bull Hosp Jt Dis 1997;56(01):63-72

10 Einhorn TA. Enhancement of fracture-healing. J Bone Joint Surg Am 1995;77(06):940-956
11 Hur H, Lee JK, Jang JW, Kim TS, Kim SH. Is it feasible to treat unstable hangman's fracture via the primary standard anterior retropharyngeal approach? Eur Spine J 2014;23(08): 1641-1647

12 Yukawa Y, Kato F, Ito K, et al. Placement and complications of cervical pedicle screws in 144 cervical trauma patients using pedicle axis view techniques by fluoroscope. Eur Spine J 2009;18 (09):1293-1299

13 Watanabe Y, Matsushita T, Bhandari M, Zdero R, Schemitsch EH Ultrasound for fracture healing: current evidence. J Orthop Trauma 2010;24(Suppl 1):S56-S61

14 Cook SD, Salkeld SL, Mse, Patron LP, Ryaby JP, Whitecloud TS. Lowintensity pulsed ultrasound improves spinal fusion. Spine J 2001; 1(04):246-254

15 Hui CF, Chan CW, Yeung HY, et al. Low-intensity pulsed ultrasound enhances posterior spinal fusion implanted with mesenchymal stem cells-calcium phosphate composite without bone grafting. Spine 2011;36(13):1010-1016 